\title{
As relações entre equipes, suporte social e bem-estar subjetivo
}

\author{
Maysa Sangy Guedes Oliveira* \\ Manuel Morgado Rezende**
}

\begin{abstract}
Resumo
Este estudo teve como objetivo investigar o papel moderador de potência de equipes sobre as relações entre percepção de suporte social e bem-estar subjetivo, mediante delineamento longitudinal. Participaram da pesquisa 249 adolescentes escoteiros, provenientes de 53 equipes. O estudo foi realizado nas cidades de Contagem e Belo Horizonte, Minas Gerais. Os resultados demonstraram que a percepção de suporte social e a potência de equipes influenciaram positiva e significativamente o bem-estar subjetivo dos adolescentes. Porém, a potência de equipes não foi capaz de moderar a relação entre percepção de suporte social e bem-estar subjetivo. Assim, os adolescentes desfrutavam de bem-estar e saúde psíquica, condição que se manteve ao longo do tempo. Tais evidências asseguram o papel promotor de bem-estar subjetivo tanto de percepção de suporte social quanto de potência de equipes na saúde mental do indivíduo. Palavras-chave: equipes; suporte social; bem-estar subjetivo; adolescentes.
\end{abstract}

\section{Relations between teams, social support and subjective well-being}

\begin{abstract}
This research had the purpose to investigate the moderating role of team potency on the relationship between social support perception and subjective well-being among scout teenagers through longitudinal delineation. This research had the participation of 249 teenagers from 53 teams. The study was carried out in the cities of Contagem and Belo Horizonte, Minas Gerais. The results demonstrated that the social support perception and the team potency influenced positively and significantly the teenager subjective welfare, however, team potency was not able to moderate the relationship between social support perception and subjective welfare. Therefore, they enjoyed well-being and mental health, condition that has remained over time. Such evidence assure the role promoter of subjective well-being as well as social support and team potency in individual's mental health.
\end{abstract}

Keywords: teams; social support; subjective well-being; teenagers.

* Doutoranda e Mestre pela UMESP, psicóloga pela PUC Minas Gerais e especialista em Administração de Empresas com ênfase em Gestão de Recursos Humanos pela Fundação Getúlio Vargas.

** Doutor pela UNICAMP, Mestre e psicólogo pela PUC Campinas, Pós-doutor em Psicologia da Saúde pela Universidade do Algarve. Professor do Programa de Pós-Graduação em Psicologia da Saúde da UMESP. 


\section{Introdução}

Quando se fala em ações dos serviços direcionados à saúde física e psicológica do adolescente, ainda há a tendência de focar nos comportamentos de risco, negligenciando outros fatores que integram o conceito de saúde. As ações direcionadas somente em conduta-problema não são suficientes para ajudar os jovens a construir suas vidas. Estudos focados no comportamento de risco serão importantíssimos, pois também colaboram para estratégias que visam à saúde dos jovens. Mas, a adolescência também possui momentos de alegria, felicidade, prazer, criatividade, construção, trocas afetivas, etc. Perceber o adolescente apenas pelo viés da fragilidade e do problema é negar suas potencialidades. Logo, pesquisas que visam aspectos sadios, positivos que previnem e promovem a saúde aos adolescentes, revelam-se como um campo promissor, atual e desafiante para a ciência.

A psicologia da saúde tem investigado e discutido temas relacionados à promoção da saúde, à prevenção e ao tratamento de doenças (Ribeiro, 2007). Neste sentido, seus construtos contribuem para estudos que buscam fortalecer os aspectos sadios do desenvolvimento humano.

O objetivo deste estudo foi investigar as relações entre potência de equipes, percepção de suporte social e bem-estar subjetivo em adolescentes escoteiros. As três variáveis estudadas pela psicologia da saúde, em sua peculiaridade, abordam questões essenciais ao adolescente: relacionamento interpessoal, saúde e bem-estar.

Foi realizada uma investigação quantitativa com aplicação de análise multivariada. É também de caráter inédito por explorar o papel moderador de potência de equipes sobre as relações entre percepção de suporte social e bem-estar subjetivo em um esboço longitudinal e relacionar as variáveis estudadas em adolescentes escoteiros.

\section{Bem-estar subjetivo}

Bem-estar subjetivo (BES) é considerado um campo de estudo da psicologia da saúde que investiga a avaliação que as pessoas fazem de suas vidas. Tal avaliação está relacionada à satisfação ou insatisfação que o indivíduo tem com sua própria vida e à frequência com que experimenta emoções positivas ou negativas (Diener \& Suh, 1997).

O conceito surgiu a partir de estudos que buscavam indicadores acerca da qualidade de vida para avaliar as mudanças sociais e implantação de políticas sociais no final da década de 1950 (Land, 1975).

De acordo com Diener (1984) o bem-estar subjetivo (BES) é considerado um construto psicológico constituído por duas dimensões associadas: satisfação com a vida (dimensão cognitiva; afetos positivos e afetos negativos (dimensão emocional). Tal visão teve como alicerce os estudos iniciais de Bradburn (1969) e de Campbell, Converge e Rodgers (1976).

Satisfação geral com vida: é baseada em um julgamento cognitivo que a pessoa faz de sua própria vida. Tal julgamento se dá através de uma comparação entre um padrão que o indivíduo estabelece e almeja para si e as circunstâncias vivenciadas (Albuquerque \& Tróccoli, 2004).

Afetos positivos e afetos negativos: compreendem as respostas de cunho afetivo, são as emoções expressas pelo indivíduo, tais como alegria, medo, etc. Fredrickson (1998) indica que as emoções positivas além de proteger, promover saúde e bem-estar a uma pessoa, são também fonte de estímulo para que ela possa florescer e crescer psicologicamente. A vida é permeada por sensações positivas e negativas, mas torna-se importante identificar se a pessoa experimentou mais emoções prazerosas do que de sofrimento (Thomas \& Diener, 1990).

O estudo de Poletto (2011), averiguou o bem-estar subjetivo e suporte social em um estudo longitudinal com crianças e adolescentes em situação de vulnerabilidade social, revelou que a vivência de afetos positivos apareceu nos resultados associada à satisfação com o self, com a família e com as amizades. Também demostrou que o suporte social as auxiliou construir respostas mais adequadas para lidar com situações adversas, permitindo crescimento psicológico e promoção da saúde física e mental.

Em síntese, um nível alto de bem-estar subjetivo só é possível através da satisfação com a vida e um número maior de emoções positivas do que negativas (Diener \& Suh, 1997).

\section{Suporte social}

Suporte social é a crença que o indivíduo tem em receber ajuda de sua rede social, que são fontes de recursos afetivos e de pertencimento. Existem três classes de crenças que auxiliam o indivíduo a processar sua percepção de suporte social: a crença de que há pessoas que o amam e se preocupam com ele; a de que é apreciado e valorizado e a de que faz parte de uma rede social (Cobb, 1976).

Este conceito surgiu na década de 1970 em estudos que investigavam como a saúde física e psicológica eram impactadas pelas relações sociais. Teve como precursores Cassel (1976) e Cobb (1976).

Em seus estudos acerca do suporte social, Kaplan, Cassel e Gore (1977), reforçam que suporte social é um fator importante na proteção contra as doenças físicas 
e psicológicas. Contudo, seu papel é coadjuvante. Além do suporte social são necessários outros significantes subjetivos que possam auxiliar o indivíduo a mobilizar seus próprios recursos no enfrentamento das doenças e problemas emocionais. Cohen e Wills (1985) defendem que a integração do indivíduo em redes sociais traz sentimentos de estabilidade e autoestima, influenciando no seu bem-estar.

Rodriguez e Cohen (1998) afirmam que as três dimensões mais estudadas que constituem o suporte social são: suporte emocional (ex: oferecer cuidado, atenção, amor a uma pessoa), suporte instrumental (fazer alguma coisa prática por alguém, ex: ajudar nas tarefas domésticas) e suporte informacional (ex: informações proporcionadas a uma pessoa no enfrentamento de um problema).

A pesquisa de Peres (2010), avaliou a relação entre suporte social e depressão em mães adolescentes, evidenciou que o suporte social se relacionou positivamente com a diminuição da depressão, ansiedade e no aumento da satisfação com a vida dessas jovens mães.

\section{Potência de equipes}

A potência de equipes é um fenômeno que surge por meio da construção social, pois consiste na crença compartilhada dos membros de uma equipe de que a mesma pode ser efetiva em seu desempenho (Shea \& Guzzo, 1987a). Portanto, trata-se de uma cognição compartilhada, uma crença coletiva. As cognições compartilhadas são fontes de probabilidades para compreender como os membros de equipes interagem e se tornam efetivos, podendo também ser utilizadas para identificar e resolver problemas ligados ao desempenho. Deste modo, o compartilhamento de conhecimentos, atitudes, crenças, valores e sentimentos são fundamentais para a formação das cognições compartilhadas (Cannon-Bowers $\&$ Salas, 2001).

Um dos estudos pioneiros referentes às crenças de grupos com atuação voltada para a eficácia foi o de Sayles (1958), que investigou a eficácia do desempenho de equipes de trabalho em uma fábrica. Seus estudos assinalaram questões fundamentais para a compreensão atual de potência em equipes, a saber: os grupos diferem de acordo com a crença coletiva de seus membros sobre sua possível eficácia; esta crença está relacionada com a eficácia real como causa e consequência e, finalmente, esta crença é moldada pelo contexto em que o grupo atua(Guzzo, Yost, Campbell \& Shea,1993).

Os estudos sobre efetividade de equipes de Shea e
Guzzo (1987a, 1987b) contribuíram de forma relevante para a ampliação do entendimento sobre o desempenho de grupos. Para os autores, a efetividade ocorre, principalmente, em função da interação entre a interdependência de tarefas, a interdependência de resultados e a potência de equipes. Mas a efetividade é também influenciada pelo ambiente no qual a equipe está inserida.

Para esse estudo, interessa a terceira variável dos estudos de Shea e Guzzo (1987a 1987b), potência de equipes, definida por eles como a crença coletiva (compartilhada) dos membros de uma equipe de que a mesma pode executar suas tarefas com efetividade.

Trata-se de um construto do nível de equipes, pois supõe que a crença na efetividade seja compartilhada por todos os membros. De acordo com os autores, a potência exerce influência sobre a efetividade e vice e versa. A crença dos membros na efetividade do grupo faz aumentar a efetividade do grupo. O senso de potência também está relacionado a experiências anteriores da equipe, sejam elas de sucesso ou de fracasso em consonância com Sayles (1958).

Além da vivência prévia de efetividade, alguns fatores internos ao grupo (objetivos do grupo, tamanho do grupo, habilidade dos membros, etc.) e externos (suporte, aprendizagem vicária, liderança da equipe, etc.), também determinam o senso de potência (Guzzo, Yost, Campbell \& Shea,1993). Os fatores externos afetam o senso de potência e estão atrelados ao sistema social do qual a equipe faz parte.

O estudo de Borba (2007), que teve como objetivo desenvolver e validar um instrumento para mensurar a potência em equipes de trabalho, demonstrou que as equipes investigadas compartilhavam internamente a crença em seu potencial, o que facilitava o bom desenvolvimento das tarefas e relações sociais favoráveis.

\section{Método}

É um estudo quantitativo com uma investigação com delineamento longitudinal e aplicação de análise multivariada.

O estudo longitudinal é um método de pesquisa que se destina a analisar um processo ao longo do tempo para investigar se ocorreram mudanças entre os períodos avaliados. Pode ser utilizado com apenas um indivíduo, com grupos, populações, organizações, etc. Diversas ciências como a Psicologia, a Sociologia e a Economia utilizam estudos longitudinais. Todavia, as ciências da saúde, em especial a Medicina (principalmente a Epidemiologia), destacam-se quanto ao número de pesquisas longitudinais. 
Para o autor, é importante que pesquisadores tenham aportes teórico-metodológicos que expliquem as mudanças observadas no desenvolvimento dos indivíduos observados ao longo do tempo. Uma das desvantagens deste método refere-se ao fato de os sujeitos investigados estarem subordinados a vieses oriundos de fatores extrínsecos, o que pode mudar o grau de comparabilidade entre os dados coletados. Outra desvantagem refere-se à perda de sujeitos ao longo da pesquisa (Haddad, 2004).

Uma vez que os processos sociais se tornam cada vez mais complexos, a informação longitudinal faz-se ainda mais necessária, principalmente nos estudos causais sobre o comportamento individual, pois podem mostrar a natureza do crescimento, medir e traçar padrões de mudança e, possivelmente, permitir interpretações causais mais fortes (Rajulton, 2001).

\section{Participantes}

Este estudo contou com a participação voluntária de 249 adolescentes escoteiros, procedentes de 53 equipes, cada uma composta por três a sete membros. O estudo foi realizado em grupos de escoteiros com sedes localizadas nas cidades de Contagem e Belo Horizonte, Minas Gerais. A idade média dos participantes foi de 13,85 anos (DP=1,86), sendo 57,4\% do sexo masculino. A escolaridade variou entre o nível fundamental incompleto $(54,6 \%)$ a médio completo $(2,40 \%)$. O tempo como membro de escotismo teve média de 3,88 anos (DP=2,37). Para ser incluído no estudo, o adolescente deveria ter no mínimo seis meses de participação no escotismo.

\section{Instrumentos}

Para a coleta de dados foi utilizado um questionário de autopreenchimento, composto por três escalas para aferir as três variáveis deste estudo, descritas abaixo:

Escala de Potência de Equipes - escala construída e validada por Guzzo (1993) e adaptada e validada para o Brasil por Siqueira, Martins, Costa e Abad (2014), composta por dez itens (alfa $=0,85)$.

Escala de Percepção de Suporte Social (EPSS) - medida construída e validada por Siqueira (2008) para aferir a dimensão de suporte prático (19 itens, alfa $=0,91)$ e a de suporte emocional (dez itens, alfa $=0,92)$.

Escalas de Bem-estar Subjetivo - para avaliar os afetos positivos e negativos foi aplicada a Escala de Animo Positivo e Negativo, construída e validada por Siqueira, Martins e Moura (1999), composta por 14 itens distribuídos em dois fatores: afetos positivos (seis itens, alfa $=0,87$ ) e afetos negativos (oito itens, alfa $=0,88)$. A Escala Satisfação Geral com a Vida, construída e validada por Siqueira, Gomide Junior e Freire (1996), foi aplicada na versão reduzida $(15$ itens, alfa $=0,84)$ para aferir a dimensão cognitiva de bem-estar subjetivo.

Ao final do questionário, para caracterização da amostra, foram acrescidas perguntas de cunho sócio demográficas.

\section{Procedimentos}

Foram apresentados a todos os envolvidos os objetivos da pesquisa. Após aceite, aprovação do Comitê de Ética e Pesquisa e preenchimento dos termos de consentimento e assentimento, a pesquisadora iniciou a coleta de dados.

Por se tratar de um estudo longitudinal, a coleta de dados foi programada em duas etapas T1 (Tempo 1) e T2 (Tempo 2), com intervalo de seis meses. Tanto no T1 quanto no T2 à pesquisadora pessoalmente aplicou o mesmo questionário com escalas que mensuravam as três variáveis do estudo. Percepção de suporte social e bem-estar subjetivo foram avaliados no nível individual. Potência de equipes também foi avaliada no individual, mas com referência à equipe de escotismo à qual o adolescente fazia parte.

Os dados coletados, todos representados por indicadores numéricos, formaram um banco de dados e foram analisados em software de análises estáticas, e submetidos às análises descritivas e multivariáveis.

As hipóteses (Quadro 1) foram formuladas levando-se em consideração as orientações de Baron e Kenny (1986) sobre estudos acerca de moderação. Segundo os autores, deve-se inicialmente investigar o impacto da variável independente sobre a dependente, na sequência o impacto da variável moderadora sobre a dependente e, por fim, o impacto da variável moderadora sobre a relação entre a variável independente e a dependente. O bem-estar subjetivo (BES) foi analisado neste estudo como variável dependente. Consequentemente, nas hipóteses (Figura 1), as três dimensões de BES também foram mencionadas como dependentes. A percepção de suporte social (PSS) foi introduzida nas hipóteses como independente. Já a potência de equipes assumiu o papel de moderadora.

\section{Resultados e Discussão}

As análises de regressão múltipla podem ser executadas de três formas: a regressão padrão, a regressão hierárquica e a stepwise. Neste estudo foi escolhido o método 


\begin{tabular}{|c|c|c|}
\hline Objetivos & Hipóteses & $\begin{array}{c}\text { Provas } \\
\text { estatísticas }\end{array}$ \\
\hline $\begin{array}{l}\text { Objetivo1: } \\
\text { Submeter aos testes } \\
\text { empíricos hipóteses acerca } \\
\text { do papel moderador de } \\
\text { potência de equipes sobre } \\
\text { as relações entre percepção } \\
\text { de suporte social e bem- } \\
\text { estar subjetivo. }\end{array}$ & $\begin{array}{l}\text { H1 - Percepção de suporte social (PSS) tem impacto positivo e significativo } \\
\text { sobre bem-estar subjetivo (BES). } \\
\text { • H1a - PSS tem impacto positivo e significativo sobre os afetos positivos. } \\
\text { - H1b - PSS tem impacto negativo e significativo sobre os afetos } \\
\quad \text { negativos. } \\
\text { - H1c - PSS tem impacto positivo e significativo sobre a satisfação geral } \\
\quad \text { com a vida. } \\
\text { H2 - Potência de equipes (PE) tem impacto positivo e significativo sobre } \\
\text { bem-estar subjetivo (BES). } \\
\text { - H2a - PE tem impacto positivo e significativo sobre os afetos positivos. } \\
\text { - H2b - PE tem impacto negativo e significativo sobre os afetos negativos. } \\
\text { - H2c - PE tem impacto positivo e significativo sobre a satisfação geral } \\
\text { com a vida. } \\
\text { H3 - Potência de equipes (PE) modera o impacto de percepção de suporte } \\
\text { social (PSS) sobre bem-estar subjetivo (BES). } \\
\text { - H3a - PE modera o impacto de PSS sobre os afetos positivos. } \\
\text { - H3b - PE modera o impacto de PSS sobre os afetos negativos. } \\
\text { - H3c - PE modera o impacto de PSS sobre a satisfação geral com a vida. }\end{array}$ & $\begin{array}{c}\text { Análises de } \\
\text { regressão } \\
\text { linear múltipla } \\
\text { hierárquica }\end{array}$ \\
\hline $\begin{array}{l}\text { Objetivo 2: } \\
\text { Testar hipóteses acerca de } \\
\text { alterações ocorridas entre o } \\
\text { Tempo } 1 \text { (T1) e o Tempo } 2 \\
\text { (T2) no papel moderador de } \\
\text { potência de equipes quanto } \\
\text { à relação entre percepção de } \\
\text { suporte social e bem-estar } \\
\text { subjetivo. }\end{array}$ & $\begin{array}{l}\text { H4 - No T2 potência de equipes (PE) aumenta o seu papel moderador } \\
\text { da relação entre percepção de suporte social (PSS) e bem-estar subjetivo } \\
\text { (BES) quando comparado a T1. } \\
\text { - H4a - No T2 PE aumenta o seu papel moderador da relação entre PSS } \\
\text { e afetos positivos quando comparado a T1. } \\
\text { - H4b - No T2 PE aumenta o seu papel moderador da relação entre PSS } \\
\text { e afetos negativos quando comparado a T1. } \\
\text { - H4c - No T2 PE aumenta o seu o seu papel moderador da relação entre } \\
\text { PSS e satisfação geral com a vida quando comparado a T1. }\end{array}$ & $\begin{array}{c}\text { Análises de } \\
\text { regressão } \\
\text { linear múltipla } \\
\text { hierárquica }\end{array}$ \\
\hline
\end{tabular}

Figura 1 - Objetivos, hipóteses e provas estatísticas aplicadas aos dados do estudo.

de regressão múltipla hierárquica, visto que ele permite testar a capacidade preditiva das variáveis independentes ao se posicionar cada uma em blocos específicos. Adicionalmente, essa técnica permite ao pesquisador controlar o efeito de uma variável independente para melhor identificar o efeito de outra variável independente, bem como identificar o efeito interativo de duas variáveis independentes na predição de uma dependente (Pallant, 2007).

Nas análises de regressão hierárquica moderadora, primeiramente foram calculados modelos com vistas a testar as hipóteses condizentes ao objetivo 1 (submeter aos testes empíricos hipóteses acerca do papel moderador de potência de equipes sobre as relações entre suporte social e bem-estar subjetivo).

\section{Análises de Regressão Hierárquica Moderadora (ARHM) no tempo 1}

Foram calculados três modelos de ARHM no Tempo 1 (T1), cada um estruturado em três blocos para cada uma das três variáveis dependentes relativas ao objetivo 1. Seguindo Cohen e Cohen (1983), entraram no primeiro bloco duas variáveis controle (idade e tempo de escotismo), as quais não foram previamente consideradas independentes, mas que poderiam deter algum valor explicativo das três dimensões de bem-estar subjetivo (BES) referidas como variáveis dependentes deste estudo. Os autores recomendam introduzir na regressão termos interativos compostos pela multiplicação da variável independente pela variável moderadora. No bloco 2 entrou 
a variável independente Percepção de Suporte Social (PSS) e a variável moderadora Potência de Equipes (PE) e no bloco 3 os termos interativos (PSSxPE), neste caso usando-se os escores centrados.

\section{Afetos Positivos (T1)}

Na primeira coluna da Tabela 1 incluiu-se o Modelo 1 com os resultados da ARHM no Tempo 1 (T1) para Afetos Positivos, dimensão emocional de BES, revelando um valor significativo de $10,2 \%$ de explicação $\left(R^{2}=0,102\right.$; $\mathrm{F}(5,243)=6,661 ; \mathrm{p}<0,01)$. No bloco 1 , calculado com as variáveis de controle idade e tempo de escotismo, foi obtido um Beta negativo e significativo $(\beta=-0,174$; $\mathrm{p}<0,05)$ apenas para idade. Estes resultados demonstram que quanto mais idade possuem esses adolescentes, menos frequentemente vivenciam tais emoções, em consonância com Silva, Matos e Diniz (2010), que também perceberam que o aumento da idade correspondeu à diminuição do BES em adolescentes.

No bloco 2 (Modelo 1 - T1) entraram a variável independente percepção de suporte social (PSS) e a variável moderadora potência de equipes (PE), onde apareceram valores significativos de Beta positivo para PSS $(\beta=0,229 ; \mathrm{p}<0,01)$ e PE $(\beta=0,129 ; \mathrm{p}<0,05)$. Tais resultados permitem confirmar as hipóteses $\mathrm{H} 1$ a e $\mathrm{H} 2 a$ (PSS e PE têm impactos positivos e significativos sobre os afetos positivos). É possível afirmar que o suporte percebido bem como a confiança de que equipe e capaz age de forma a fortalecer a frequência com que ocorrem experiências emocionais positivas entre os adolescentes escoteiros confirmando os achados dos estudos de Cohen e Wills (1985) e Peres (2010).

No último bloco do Modelo 1 (T1), foram introduzidos os valores interativos (PSSxPE), sendo uma independente (PSS) e outra moderadora (PE). O Beta dos termos interativos indicou impacto negativo e não significativo $(\beta=-0,118 ; \mathrm{NS})$, o que não confirmou a hipótese H3a (PE modera o impacto de PSS sobre os afetos positivos). Verifica-se que potência de equipes não consegue moderar, aumentar nem diminuir o impacto de percepção de suporte social sobre afetos positivos.

\section{Afetos Negativos (T1)}

Estão contidos no Modelo 2 do Tempo 1 (T1) os resultados das ARHM no T1 para Afetos Negativos, dimensão emocional de BES, que explicou apenas $2,6 \%\left(\mathrm{R}^{2}=\right.$ $0,026 ; \mathrm{F}(5,243)=2,313 ; \mathrm{p}<0,05)$ da sua variância. No primeiro bloco estão os resultados dos Betas para aferir as variáveis de controle idade $(\beta=0,074 ; \mathrm{NS})$ e tempo de escotismo ( $\beta=-0,029 ; \mathrm{NS})$, não tendo sido obtidos valores significativos de predição para afetos negativos (Tabela 1) Portanto, a idade e o tempo no escotismo não exerceram nenhuma influência sobre a frequência das emoções negativas dos adolescentes deste estudo.

No segundo bloco do Modelo 2 no T1 (Tabela 1) ao se introduzir a variável independente PSS e a moderadora

\begin{tabular}{|c|c|c|c|}
\hline Variáveis & $\begin{array}{c}\text { Modelo 1 } \\
\text { Afetos } \\
\text { positivos } \\
\beta \\
\end{array}$ & $\begin{array}{c}\text { Modelo } 2 \\
\text { Afetos } \\
\text { negativos } \\
\beta\end{array}$ & $\begin{array}{c}\text { Modelo } 3 \\
\text { Satisfação geral } \\
\text { com a vida } \\
\beta\end{array}$ \\
\hline \multicolumn{4}{|l|}{ Bloco 1: variáveis de controle } \\
\hline Idade & $-0,174 *$ & 0,074 & $-0,150 *$ \\
\hline Tempo de escotismo & 0,063 & $-0,029$ & $0,126 *$ \\
\hline \multicolumn{4}{|l|}{ Bloco 2: variável independente e moderadora } \\
\hline Percepção de suporte social (PSS) & $0,229 * *$ & $-0,044$ & $0,259 * *$ \\
\hline Potência de equipes (PE) & $0,129 *$ & $-0,178^{*}$ & $0,265^{* *}$ \\
\hline \multicolumn{4}{|l|}{ Bloco 3: termos interativos } \\
\hline $\begin{array}{l}\text { Percepção de suporte social x Potência de equipes } \\
\text { (PSSxPE) }\end{array}$ & $-0,118$ & 0,062 & 0,015 \\
\hline F modelo & $6,661 * *$ & $2,313^{*}$ & $12,196^{* *}$ \\
\hline $\mathbf{R}^{2}$ modelo & 0,102 & 0,026 & 0,184 \\
\hline$\%$ de variância explicada pelo modelo & 10,2 & 2,6 & 18,4 \\
\hline
\end{tabular}

Tabela 1 - Modelos de análise de regressão hierárquica moderadora (ARHM) para afetos positivos, afetos negativos e satisfação geral com a vida no T1 ( $\mathrm{n}=249)$ 
PE, observou-se um valor significativo e negativo de Beta apenas para $\mathrm{PE}(\beta=-0,178 ; \mathrm{p}<0,01)$. Mediante tais resultados não se confirmou a hipótese H1b (PSS tem impacto negativo e significativo sobre os afetos negativos). Por outro lado, permitiram sustentar a $H 2 b$ (PE tem impacto negativo e significativo sobre os afetos negativos).

No terceiro bloco do Modelo 2 no T1 está o valor não significativo e negativo de Beta $(\beta=-0,062$ : NS) dos valores interativos PSSxPE (Tabela 1), o que não sustentou a hipótese $H 3 b$ (PE modera o impacto de PSS sobre os afetos negativos).

Logo, os resultados confirmaram que, nem a idade, nem o tempo de escotismo, muito menos a percepção de suporte social, foram capazes de interferir ou mesmo diminuir os afetos negativos dos adolescentes, o que foi conseguido somente pela potência de equipes. Parece provável que o tempo que os adolescentes dedicam às suas atividades em equipes dentro do escotismo torna-se fator importante para minimizar seus afetos negativos.

\section{Satisfação geral com a vida (T1)}

O Modelo 3, calculado para a variável Satisfação geral com a vida (SGV) no T1 (Tabela 1), conseguiu explicar $18,4 \%$ da variância $\left(\mathrm{R}^{2}=0,184: \mathrm{F}(5,243)=12,196\right.$; $\mathrm{p}<0,01)$. No bloco 1 as variáveis 'idade' e 'tempo de escotismo' inseridas apenas como controle produziram Betas significativos, cujos valores foram, respectivamente, negativos para idade $(\beta=-0,150 ; \mathrm{p}<0,05)$ demonstrando que a satisfação com a vida desses adolescentes também diminui quando a idade aumenta. Em relação ao tempo de escotismo $(\beta=0,126 ; p<0,05)$, o resultado foi positivo, quanto mais tempo eles se relacionam com o escotismo, maior é o nível de satisfação com a vida.

Ainda no Modelo 3 (T1), no segundo bloco (Tabela 1), acrescido da variável independente percepção de suporte social e da variável moderadora potência de equipes, foram obtidos dois Betas positivos e significativos. Um referente à PSS $(\beta=0,259: \mathrm{p}<0,01)$ e o outro para PE $(\beta=$ $0,265 ; \mathrm{p}<0,01)$. Tais resultados permitem confirmar as hipóteses H1c e H2c(PSS e PE têm impactos positivos e significativos sobre a SGV). Deste modo, os momentos que esses jovens partilham das atividades em equipe dentro do escotismo parece trazer elementos positivos também em relação à satisfação com a vida.

No último bloco do Modelo 3 do T1 (Tabela 1), com presença dos valores interativos (PSSXPE) das duas variáveis, revelou-se um valor positivo e não significativo de Beta $(\beta=0,015$; NS). Estes resultados não sustentam a hipótese $H 3$ c (a capacidade moderadora de PE sobre a relação entre PSS e SGV). Parece adequado afirmar, que

\begin{tabular}{|c|c|c|c|}
\hline Variáveis & $\begin{array}{c}\text { Modelo } 1 \\
\text { Afetos } \\
\text { positivos } \\
\beta\end{array}$ & $\begin{array}{c}\text { Modelo } 2 \\
\text { Afetos } \\
\text { negativos } \\
\beta\end{array}$ & $\begin{array}{c}\text { Modelo } 3 \\
\text { Satisfação } \\
\text { geral com a } \\
\text { vida } \\
\beta\end{array}$ \\
\hline \multicolumn{4}{|l|}{ Bloco 1: variáveis de controle } \\
\hline Idade & $-0,206^{* *}$ & 0,021 & $-0,125^{*}$ \\
\hline Tempo de escotismo & 0,098 & $-0,018$ & $0,129 *$ \\
\hline Potência de equipe no T1 (PE T1) & $-0,109$ & $-0,002$ & 0,011 \\
\hline \multicolumn{4}{|c|}{ Bloco 2: variável independente e moderadora } \\
\hline Percepção de suporte social (PSS2) & $0,330^{* *}$ & $-0,088$ & $0,285^{* *}$ \\
\hline Potência de equipes (PE2) & $0,208^{*}$ & $-0,131$ & $0,308^{* *}$ \\
\hline \multicolumn{4}{|l|}{ Bloco 3: termos interativos } \\
\hline PSS2x PE2 & 0,094 & 0,070 & 0,045 \\
\hline F modelo & $4,579 * *$ & 1,068 & $3,247 * *$ \\
\hline $\mathbf{R}^{2}$ modelo & 0,173 & 0,018 & 0,235 \\
\hline$\%$ de variância explicada pelo modelo & 17,3 & 1,8 & 23,5 \\
\hline
\end{tabular}

${ }^{*} \mathrm{p}<0,05: * * \mathrm{p}<0,01$

Tabela 2 - Modelos de análise de regressão hierárquica moderadora (ARHM) para afetos positivos, afetos negativos e satisfação geral com a vida no T2 $(n=249)$. 
o impacto positivo exercido pela percepção de suporte social sobre o nível de satisfação com a vida dos adolescentes não pode ser alterado pela crença dos mesmos sobre a eficácia de sua equipe.

Há que se considerar que os resultados da ineficácia de moderação de $P E$ sobre a relação entre $P S S$ e BES podem revelar: ainda que a convicção mantida pelos adolescentes sobre a eficácia de sua equipe se manifeste como fator positivo, proporcionando-lhes satisfação com a vida e mais sentimentos positivos, sua força não é tão grande a ponto de interferir na satisfação que expressam pelo suporte percebido. Tais resultados também fortalecem a importância do suporte social no bem-estar das pessoas, sobretudo quando integradas a grupos tidos como positivos, conforme estudos anteriores, com o de Kaplan, Cassel e Gore (1977) e Poletto (2011).

\section{Análises de Regressão Hierárquica Moderadora (ARHM) no Tempo 2}

Como este estudo teve um delineamento longitudinal, na segunda fase, Tempo 2 (T2) das análises de regressão múltipla hierárquica moderadora (ARHM) foram calculados modelos com vistas a testar as hipóteses condizentes ao objetivo 2 (testar hipóteses acerca de alterações ocorridas entre o T1 e o T2 no papel moderador de potência de equipes na relação entre percepção de suporte social e bem-estar subjetivo).

As ARHM do T2 (Tabela 2) mais uma vez foram organizadas em três modelos constituídos, cada um por três blocos. No primeiro bloco entraram as variáveis de controle idade e tempo de escotismo, sendo necessário introduzir a variável potência de equipes do T1 visando eliminar sua possível influência nos escores de afetos positivos, afetos negativos e satisfação geral com a vida no T2; no segundo bloco entrou a variável independente percepção de suporte social no tempo 2 (PSST2) e a variável moderadora potência de equipes no tempo 2 (PET2); no terceiro bloco entraram os termos interativos no T2 (PSST2xPET2), os quais foram calculados após centralização dos escores médios.

\section{Afetos Positivos (T2)}

$\mathrm{Na}$ ARHM no Tempo 2 (T2) os Afetos Positivos (Tabela 2) receberam do Modelo 1 maior explicação $17,3 \%\left(\mathrm{R}^{2}=0,173 ; \mathrm{F}(6,242)=4,579 ; \mathrm{p}<0,01\right)$ do que no T1 $(10,2 \%)$, bem como impactos positivos e significativos da variável independente Percepção de Suporte Social $(\beta=0,330 ; p<0,01)$ e da variável moderadora Potência de Equipes $(\beta=0,208 ; \mathrm{p}<0,01)$ do que no T1. Assim, no T2 destaca-se o impacto de PSS e PE sobre a frequência das emoções positivas desses adolescentes não só se manteve, mas, aumentou.

No T2 os impactos significativos e negativos da idade também foram maiores $(\beta=-0,206 ; p<0,01)$, revelando que o aumento da idade continuou a impactar negativamente as experiências emocionais positivas desses jovens.

Quanto à hipótese H4a (no T2 PE aumenta o seu papel moderador da relação entre PSS e afetos positivos quando comparado a T1)não foi confirmada, visto que não houve aumento no valor de Beta entre o T1 e o $\mathrm{T} 2(\beta=0,094 ; \mathrm{NS})$.

\section{Afetos Negativos (T2)}

Os resultados da ARHM no Tempo 2 (T2) dos Afetos Negativos receberam do Modelo 2 (Tabela 2) menor explicação 1,8\% $\left(\mathrm{R}^{2}=0,018 ; \mathrm{F}(6,242)=1,068 ; \mathrm{NS}\right)$ quando comparado ao T1 (2,6\%). Igualmente, os impactos da idade $(\beta=0,021 ; \mathrm{NS})$, tempo de escotismo $(\beta=-0,018$; NS) e percepção de suporte social $(\beta=-0,088$; NS), bem como a potência de equipes $(\beta=-0,131$; NS) não se mostraram capazes de exercer poder sobre os afetos negativos dos adolescentes.

Deste modo, é impossível haver fortalecimento da interseção entre PE e PSS ( $\beta=0,070$; NS) ao longo do tempo, muito menos de PE ser capaz de agir como moderadora e alterar seu impacto sobre a relação entre PSS e afetos negativos. Tais evidências nos resultados não sustentam a hipótese H4b (no T2 PE aumenta o seu papel moderador da relação entre PSS e afetos negativos quando comparado a T1).

\section{Satisfação geral com a vida (T2)}

Os resultados da ARMH da variável Satisfação Geral com a Vida (SGV) no Tempo 2 (T2) evidenciaram maior explicação 23,5\% $\left(\mathrm{R}^{2}=0,235 ; \mathrm{F}(6,242)=3,247 ; \mathrm{p}<0,01\right)$ do que no T1 $(18,4 \%)$ representando um valor expressivo de sua variância (Tabela 2). Também demonstram impactos significativos e positivos da variável percepção de suporte social $(\beta=0,285 ; \mathrm{p}<0,01)$ e potência de equipes $(\beta=0,308 ; p<0,01)$ do que no T1 (Tabela 2$)$.

Desta forma, mesmo com a passagem do tempo receber apoio de uma rede social e pertencer a uma equipe percebida como competente continuo a auxiliar os jovens a avaliar positivamente a vida. Garantindo o papel promotor de saúde de BES, PSS e PE.

Quanto à idade e tempo de escotismo, no T2 os resultados comprovaram impactos significativos e negativos $(\beta=-0,125 ; p<0,05)$, confirmando que o avanço da 
idade continuo a diminuir a satisfação com a vida destes jovens. Entretanto, ao longo do tempo, as atividades no escotismo permaneceram como uma fonte de recursos prazerosos e afetivos para eles.

Sobre o papel moderador, percebeu-se um valor de Beta não significativo $(\beta=0,045$; NS), não sendo suficiente confirmar a hipótese $H 4 c$ (no T2 PE aumenta o seu papel moderador da relação entre PSS e SGV quando comparado a T1).

Por fim, se no T1 ficou comprovado à inexistência de moderação de PE no impacto de PSS sobre as três dimensões de BES (afetos positivos, afetos negativos e satisfação geral com a vida), conforme as hipóteses $\mathrm{H}_{3}$ a, $b$, c, seria pouco provável que no T2 as hipóteses $\mathrm{H} 4 a, b$, $c$ fossem confirmadas.

\section{Conclusão}

Esta investigação, com delineamento longitudinal, teve como objetivo principal pesquisar o papel moderador de potência de equipes sobre as relações entre percepção de suporte social e bem-estar subjetivo.

Através dos resultados das ARLM (T1 e T2), ficou demonstrado que a percepção de suporte social e a crença na eficácia da equipe promovem emoções positivas e satisfação geral com a vida dos adolescentes escoteiros. Em contraponto, a potência de equipe não consegue aumentar ou diminuir o impacto de PSS sobre as dimensões de BES. Ainda que a percepção de suporte social e potência de equipes tenham atuado em separado, ambas revelaram índices positivos e significativos de influência sobre o bem-estar subjetivo dos adolescentes escoteiros.

Este estudo contribuiu para comprovar e reforçar empiricamente que, um bom nível de bem-estar subjetivo e a percepção de receber apoio de uma rede social podem proteger e promover saúde, bem como ser uma fonte de estímulo para o crescimento psicológico de adolescentes. Igualmente, reforça pesquisas que visam instituições que ajudam a promover a saúde física e psicológica ao indivíduo conforme defendido pela psicologia da saúde.

Já os estudos sobre potência de equipes podem colaborar para pesquisas que visem à eficácia do desempenho das equipes e/ou grupos em relação às atividades, bem como a qualidade dos relacionamentos interpessoais.

O estudo também revelou algumas limitações: a primeira é que não foi possível controlar o viés nos dados fornecidos pelos participantes; a segunda refere-se ao fato das pesquisas sobre potência de equipes serem recentes; por último, por se tratar de um estudo inédito, também não foi possível comparar os resultados com pesquisas anteriores. Deste modo, sugere-se realizar outros estudos sobre as variáveis que influenciam o desempenho de equipes, entre elas, o de 'potência de equipes', inclusive os que possam comparar com os resultados deste estudo. Bem como, estudos que investiguem instituições (grupos, comunidades, movimentos, etc.) que promovam saúde psicológica e bem-estar ao indivíduo.

\section{Referências}

Albuquerque. A.S., \& Tróccoli, B.T. (2004). Desenvolvimento de Uma Escala de Bem-Estar Subjetivo. Psicologia: Teoria e Pesquisa, 20(2), 153-164.

Baron, R. M., \& Kenny, D. A. (1986) The Moderator-Mediator Variable Distinction in Social Psychological Research: Conceptual, Strategic, and Statistical Considerations. Journal of Personality and Social Psychology, 51(6), 1173-1182.

Borba, A. C. (2007) Potência em equipes: desenvolvimento de uma medida. Dissertação de Mestrado, Instituto de Psicologia, Universidade de Brasília, Brasília.

Bradburn, N. M. (1969). The structure of psychological well-being. Chicago: Aldine Publishing.

Cohen, J., \& Cohen, P. (1983). Applied multiple regression/correlation analysis for the behavioral sciences. Hillsdale, NJ: Lawrence Erlbaum Associates.

Cannon-Bowers, J. A., \& Salas, E. (2001). Reflections on shared cognition. Journal of Organizational Behavior, 22(2), 195-202.

Campbell, A., Converge, P. E., \& Rodgers, W. L. (1976). The quality of American life. New York: Russell Stage Foundation.

Cassel, J. C. (1976). The contribution of the social environment to host resistance. American Journal of Epidemiology, 104(2), 107-123.

Cobb, S. (1976). Social support as a moderator of life stress. Psychossomatic Medicine, 38(5), 300-314.

Cohen, S., \& Wills, T. A. (1985). Stress and the buffering hypothesis. Psychological Bulletin, 98(2), 310-377.

Diener, E. (1984). Subjective well being. Psychological Bulletin, 95(3), 542-575.

Diener, E., \& Suh, E. (1997). Measuring quality of life: Economic, social, and subjective indicators. Social Indicators Research, 40(1), 189-216.

Fredrickson, B.L. (1998). What good are positive emotions? Review of General Psychology, 2(3), 300-319.

Guzzo, R. A., Yost, P.R., Campbell, J., \& Shea, G. P. (1993). Potency in groups: articulating a construct. British Journal of Psychology, 32(1), 87-106.

Haddad, N. (2004). Metodologia de estudos em ciências da saúde: como planejar, analisar e apresentar um trabalho científico. São Paulo: Roca.

Kaplan, B., Cassel, J., \& Gore, S. (1977). Social support and health. Medical Care, 15(5), 47-58.

Land, K. C. (1975). Social indicators models: An overview. Em: Land, C., \& Spilerman, S. (Orgs.). Social indicator models. New York: Russell Sage Foundation, (pp.5-36).

Pallant, J. (2007). SPSS - Survival Manual. New York: Open University Press.

Poletto, M. (2011). Bem-estar subjetivo: um estudo longitudinal com crianças e adolescentes em situação de vulnerabilidade social. Tese de Doutorado, Instituto de Psicologia, Universidade Federal do Rio Grande do Sul, Porto Alegre.

Peres, A.P.A. (2010). Suporte social e depressão gestacional: avaliando a relação em uma amostra de adolescentes. Dissertação de Mestrado, Programa de Pós-Graduação em Saúde e Comportamento, Universidade Católica de Pelotas, Pelotas, Rio Grande do Sul.

Rajulton, F. (2001). The Fundamentals of Longitudinal Research: An Overview. Special Issue on Longitudinal Methodology. Canadian Studies in Population, 28(2), 169-185.

Ribeiro, J. L. P. (2007). Introdução à Psicologia da Saúde (2a ed. rev.). Coimbra: Quarteto.

Rodriguez, M. S., \& Cohen, S. (1998). Social support. Encyclopedia of Mental Health, 3, 535-544.

Sayles, L. R. (1958). The Behaviour of Industrial Work Groups. New York: Wiley. Shea, G. P., \& Guzzo, A. (1987a). Groups as human resources. Em: Rowland, M., \& Ferris, R. (Eds.). Research in Personnel and Human Resources Management. Greenwich, CT: JAI Press, (pp.323-356).

Shea, G. P., \& Guzzo, R. A. (1987b). Group effectiveness: What really matters? Sloan Management Review, 28, 25-31. 
Silva, A. B., Matos, M. G., \& Diniz, G. A. (2010). Idade, género e bem-estar subjectivo nos adolescentes. Revista Portuguesa de Pedagogia, 44(2), 39-60.

Siqueira, M. M. (2008). Construção e validação da Escala de Percepção de Suporte Social. Psicologia em Estudo, 13(2), 381-388.

Siqueira, M. M., Martins, M. C. F., Costa L. V., \& Abad, A. Z. (2014). Potência de equipes de trabalho. Em: Siqueira, M. (Org.). Novas medidas do comportamento organizacional - ferramentas de diagnóstico e de gestão. Porto Alegre: Artmed, (pp. 256-263).

Siqueira, M. M., Gomide Jr, S., \& Freire, S. A. (1996). Construção e validação de uma Escala de Satisfação Geral com a Vida (ESGV). Manuscrito não publicado, Universidade Federal de Uberlândia, Uberlândia.
Siqueira, M. M., Martins, M. C. F., \& Moura, O. I. (1999). Construção e validação fatorial da EAPN: Escala de Ânimo Positivo e Negativo. Revista da Sociedade de Psicologia do Triângulo Mineiro, 2(3), 34-40.

Thomas, D., Diener, E. (1990). Memory accuracy in the recall of emotions. Journal of Personality and Social Psychology, 59(2), 291-297.

Submetido em: 10-5-2017

Aceito em: 28-6-2017 